\title{
Exhaustion of tumor-specific CD8+ T cells in metastases from melanoma patients
}

\author{
Lukas Baitsch, ${ }^{1}$ Petra Baumgaertner, ${ }^{1}$ Estelle Devêvre, ${ }^{1}$ Sunil K. Raghav, ${ }^{2}$ \\ Amandine Legat, ${ }^{1}$ Leticia Barba, ${ }^{1}$ Sébastien Wieckowski, ${ }^{3}$ Hanifa Bouzourene, ${ }^{3}$ \\ Bart Deplancke, ${ }^{2}$ Pedro Romero, ${ }^{4}$ Nathalie Rufer, ${ }^{1,3}$ and Daniel E. Speiser ${ }^{1}$
}

\begin{abstract}
${ }^{1}$ Clinical Tumor Immune-Biology Unit, Ludwig Institute for Cancer Research, Lausanne, Switzerland. 2Laboratory of Systems Biology and Genetics, Institute of Bioengineering, School of Life Sciences, Ecole Polytechnique Fédérale de Lausanne, Lausanne, Switzerland. ${ }^{3}$ University Hospital Center and

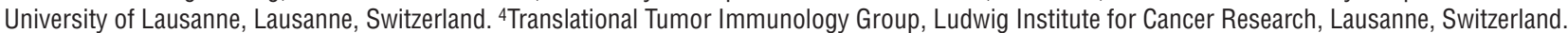

\begin{abstract}
In chronic viral infections, $\mathrm{CD8}^{+} \mathrm{T}$ cells become functionally deficient and display multiple molecular alterations. In contrast, only little is known of self- and tumor-specific $\mathrm{CD8}^{+} \mathrm{T}$ cells from mice and humans. Here we determined molecular profiles of tumor-specific $\mathrm{CD8}^{+} \mathrm{T}$ cells from melanoma patients. In peripheral blood from patients vaccinated with $\mathrm{CPG}$ and the melanoma antigen Melan-A/MART-1 peptide, we found functional effector $T$ cell populations, with only small but nevertheless significant differences in $T$ cells specific for persistent herpesviruses (EBV and CMV). In contrast, Melan-A/MART-1-specific T cells isolated from metastases from patients with melanoma expressed a large variety of genes associated with $T$ cell exhaustion. The identified exhaustion profile revealed extended molecular alterations. Our data demonstrate a remarkable coexistence of effector cells in circulation and exhausted cells in the tumor environment. Functional $\mathrm{T}$ cell impairment is mediated by inhibitory receptors and further molecular pathways, which represent potential targets for cancer therapy.
\end{abstract}

\section{Introduction}

$\mathrm{CD}^{+} \mathrm{T}$ cell responses in acute viral diseases have been extensively characterized in mice and humans (1-6). While viruses multiply rapidly during the first week of infection, $\mathrm{CD}^{+} \mathrm{T}$ cells become activated and expand vigorously, reaching a peak of $\mathrm{T}$ cell effector function. In parallel with the consequent decline of viral antigen, the majority of $\mathrm{CD}^{+} \mathrm{T}$ cells undergo apoptosis (contraction phase). After pathogen clearance, memory $\mathrm{T}$ cells persist for years at low frequencies, ready for accelerated protective immune responses in case of reinfection.

When pathogens are not eliminated, T cells may persist in much larger numbers. They are composed of large numbers of effector cells and low percentages of memory cells. Essentially, there are 2 scenarios of long-term $\mathrm{CD}^{+} \mathrm{T}$ cell activity in viral infection: the first scenario is observed, e.g., in persistent herpesvirus infection (e.g., EBV, CMV) in healthy individuals, where T cells successfully contain the viruses and thus are protective even though they do not eliminate the viruses entirely. The second scenario is associated with viral spread and progressive tissue damage in the presence of large numbers of $\mathrm{CD}^{+} \mathrm{T}$ cells, e.g., in HIV-1, HBV, or HCV infection, and in the murine model of lymphocytic choriomeningitis virus clone 13 (LCMV clone 13) infection. These 2 scenarios are distinguished by a fundamentally different functional competence of $\mathrm{CD}^{+} \mathrm{T}$ cells. In the first scenario, such as in healthy donors infected with EBV or CMV, viral antigen-specific $\mathrm{T}$ cells are functionally competent and thus ready for immediate cytokine production and cytotoxicity (7). These cells contribute to rapid reduction of viral load and restoration of health by viral containment to small anatomical compartments (8-10). In contrast, $\mathrm{CD}^{+} \mathrm{T}$ cells in the second scenario (i.e., the failure of viral containment) are functionally impaired $(11,12)$. The murine infection with LCMV

Conflict of interest: The authors have declared that no conflict of interest exists. Citation for this article: J Clin Invest. 2011;121(6):2350-2360. doi:10.1172/JCI46102. clone 13 is a prototype model of functional $\mathrm{T}$ cell impairment, called $\mathrm{T}$ cell exhaustion, with progressively reduced production of IL-2, TNF- $\alpha$, and IFN- $\gamma$, followed by incapacity to lyse (infected) target cells $(13,14)$. Analysis of such cells has led to significant discoveries, such as the identification of PD-1, a major inhibitory receptor involved in $\mathrm{T}$ cell function (15). Gene expression profiling of murine $\mathrm{T}$ cells allowed a global assessment, revealing that $\mathrm{T}$ cell exhaustion is associated with numerous molecular alterations, affecting genes regulating chemotaxis, adhesion, coreceptors, migration, metabolism, and energy (2). Hereafter, we call these multiple changes exhaustion profile.

In humans, functional deficits were found in HIV-1-, HCV-, and HBV-specific $\mathrm{CD}^{+} \mathrm{T}$ cells $(11,16,17)$, and a recent gene expression study described an exhaustion profile in HIV-1 patients (18). In contrast to virus-specific T cells, only little is known of self- and tumor-specific $\mathrm{CD}^{+} \mathrm{T}$ cells. In humans and mice, it remains to be determined whether functional impairments of bona fide self-antigen-specific $T$ cells represent exhaustion, anergy, or other functional states. In melanoma patients, there are substantial numbers of long-term persisting effector-memory $\mathrm{CD}^{+} \mathrm{T}$ cells, despite failures of immune protection from disease. Circulating human tumor-specific $\mathrm{CD}^{+} \mathrm{T}$ cells may be cytotoxic and produce cytokines in vivo (19-21), indicating that self- and tumor-specific human CD8 ${ }^{+}$ $\mathrm{T}$ cells can reach functional competence after potent immunotherapy such as vaccination with peptide, incomplete Freund's adjuvant (IFA), and CpG (19) or after adoptive transfer (22). In contrast to peripheral blood, $\mathrm{T}$ cells from metastasis are functionally deficient, with abnormally low cytokine production and upregulation of the inhibitory receptors PD-1, CTLA-4, and TIM-3 (20, 23-25). Functional deficiency is reversible, since T cells isolated from melanoma tissue can restore IFN- $\gamma$ production after short-term in vitro culture (20). However, it remains to be determined whether this functional impairment involves 
A

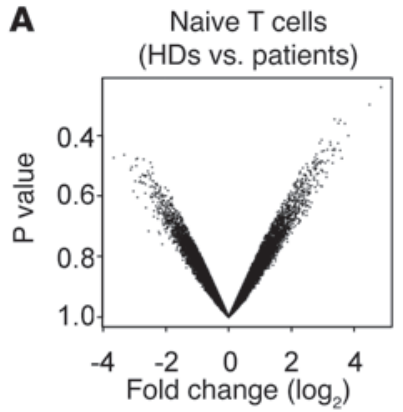

C

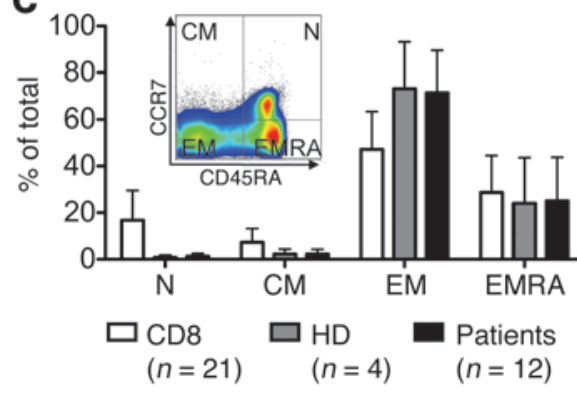

B EBV-specific T cells (HDs vs. patients)

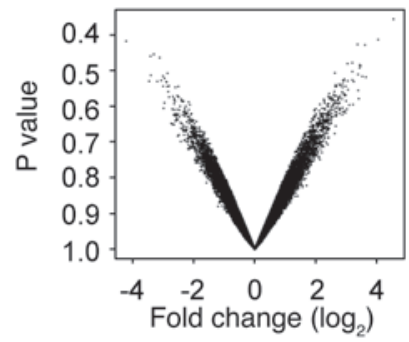

D

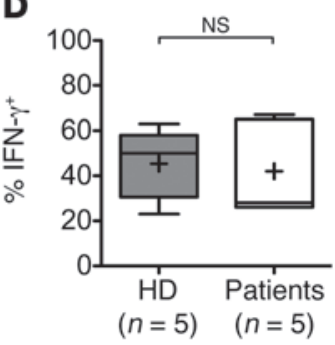

Figure 1

Naive and virus-specific $T$ cells show no significant differences between melanoma patients and healthy donors. (A and $\mathbf{B}$ ) Volcano plots for all gene probes on the microarray, showing expression differences and $P$ values of naive T cells (healthy donors [HDs] versus patients; A) or EBV-specific T cells (healthy donors versus patients; B). Each point represents 1 gene probe. (C) Lymphocytes were stained with an A2/EBV BMLF1 $1_{280-288}$ tetramer together with anti-CD8, antiCD45RA, and anti-CCR7. The inset shows a dot plot distinguishing the phenotypes among total $C D 8^{+} T$ cells analyzed as controls: naive (N) $\left(\mathrm{CD} 45 \mathrm{RA}^{+} \mathrm{CCR} 7^{+}\right)$, central memory $(C M)\left(C D 45 R A^{-} C C R 7^{+}\right)$, effector memory (EM) (CD45RA-CCR7-) and effector memory RA ${ }^{+}$cells (EMRA) (CD45RA $\left.{ }^{+} C C R 7^{-}\right)$. Bar graph depicts the percentage (mean $\pm \mathrm{SD}$ ) of each phenotype of total CD8 ${ }^{+}$ cells or total EBV tetramer-positive populations from healthy donors or patients. (D) IFN- $\gamma$ production by EBV-specific T cells upon 4-hour stimulation. Whiskers in box plots indicate maximum and minimum values measured. Cross indicates the mean, while line indicates the median. further molecular pathways, possibly resembling $\mathrm{T}$ cell exhaustion or anergy as defined in animal models $(2,26)$.

The identification of mechanisms responsible for functional impairment of self- and tumor-specific $\mathrm{T}$ cells may reveal targets for novel cancer therapies. Human $\mathrm{CD}^{+} \mathrm{T}$ cell responses specific for the melanoma antigen Melan-A/MART-1 represent a model wherein self-specific T cells can be studied in great detail. Furthermore, we took advantage of the strong immunogenicity of vaccination with peptide plus CPG (19). By direct ex vivo analysis, we compared Melan-A/MART-1-specific T cells (hereafter called tumor-specific $\mathrm{T}$ cells) with virus-specific $\mathrm{T}$ cells by microarray analysis, quantitative PCR (qPCR), and flow cytometry. Recent studies focused on circulating $T$ cells (27), whereas $T$ cells residing in tumor tissue remain poorly characterized. Therefore, we isolated $\mathrm{T}$ cells from both peripheral blood and metastases. We found that the former show molecular and functional features of effector cells, similar to circulating CMV-specific T cells, demonstrating that human self- and tumor-specific T cells have the potential to become competent effector cells. In marked contrast, the tumor-specific T cells isolated from metastatic tissue displayed an exhaustion profile, consisting of large numbers of molecular alterations.

\section{Results}

Naive and virus-specific T cells show no significant differences between melanoma patients and healthy donors. Recently we demonstrated reproducibility of gene expression profiling of small numbers of $(1,000) \mathrm{T}$ cells (28). Applying this technique (Supplemental Figure 1, A-D; supplemental material available online with this article; doi:10.1172/ JCI46102DS1), we analyzed naive and antigen-specific T cells upon sorting of PBMC subsets by flow cytometry. We compared gene expression profiles of naive $\mathrm{CD} 8^{+} \mathrm{T}$ cells from melanoma patients and healthy donors and found no significant differences (Figure 1A), confirming previous studies (29). For the isolation of antigen-specific cells, we used tetramers and sorted $T$ cells specific for the tumor antigen Melan-A/MART-1, the EBV antigen BMLF1, and the CMV antigen pp65. We compared EBV-specific T cells between healthy donors and patients and did not observe significant differences in gene expression (Figure 1B). In parallel, we found similar phenotypes and similar IFN- $\gamma$ production (Figure $1, \mathrm{C}$ and $\mathrm{D}$ ). Thus, many $\mathrm{CD}^{+} \mathrm{T}$ cells appeared relatively normal in our patients.

Gene expression profiling of naive versus nonnaive T cells. Before analyzing tumor-specific $T$ cells, we validated our approach using only 1,000 cells, by searching for the known molecular differences between naive and nonnaive $\mathrm{CD}^{+} \mathrm{T}$ cells $(28,30)$. We selected genes showing a 3 -fold or greater change between naive and nonnaive $\mathrm{CD}^{+} \mathrm{T}$ cells, plus a $P$ value adjusted for the false discovery rate (FDR) of less than 0.05 (Supplemental Figure 2A). With this strategy, we identified 409 upregulated and 364 downregulated genes in naive relative to nonnaive $\mathrm{CD}^{+} \mathrm{T}$ cells (Supplemental Table 1 ) and found that all naive $T$ cell populations clustered together and apart from all nonnaive $\mathrm{T}$ cells (Figure 2A). We selected 8 genes for verification by qPCR. Without exception, they confirmed the microarray results, whereby qPCR detected quantitatively larger differences, owing to the higher sensitivity of qPCR (Supplemental Figure 2B). Additionally, the data for many of the differentially expressed genes (e.g., CCR7, LEF1, SELL, IFNG, GZMB, and HLADR; Supplemental Figure 2C) confirmed well-known differences between naive and nonnaive $\mathrm{T}$ cells.

We assessed biological classification of the 773 differentially expressed genes by assigning them to 9 Gene Ontology (GO) terms and then determined whether any of these GO terms were overrepresented in our list compared with the predicted frequency in a random gene list. Not surprisingly, we found about twice as many immune response genes as the number predicted from a random gene test (Figure 2B). Additionally, the GO terms for translation, cell death, and apoptosis were overrepresented in nonnaive cells, whereas genes involved in DNA repair were underrepresented.

In 2005, Willinger et al. made a thorough gene expression analysis of human $\mathrm{CD}^{+} \mathrm{T}$ cells from healthy donors without distinction of antigen specificity (31). They determined large differences between naive and total effector cells, providing gene 
A

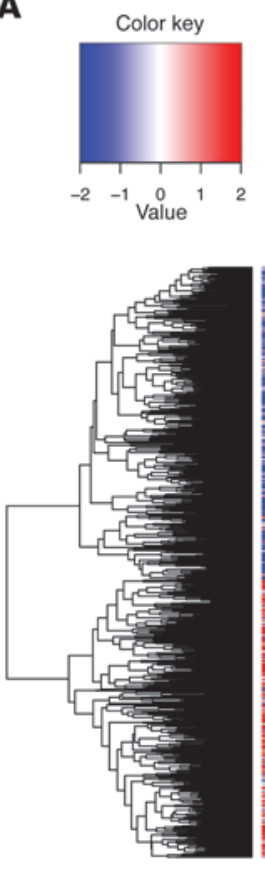

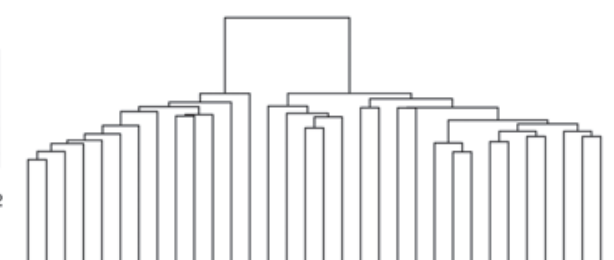

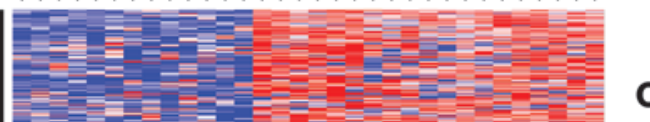

B

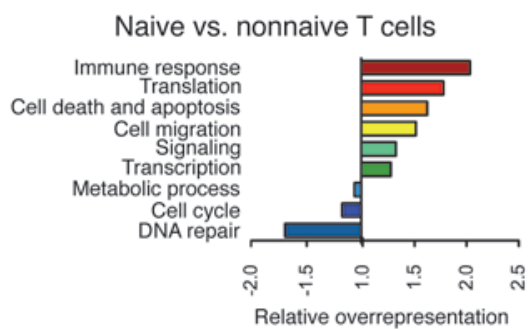

C

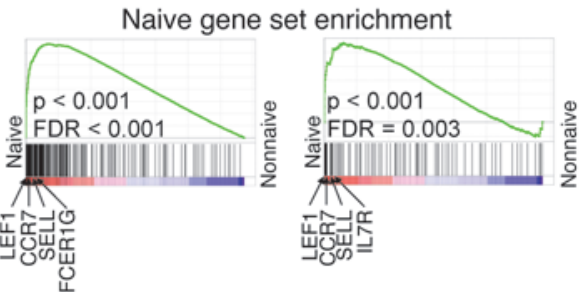

D

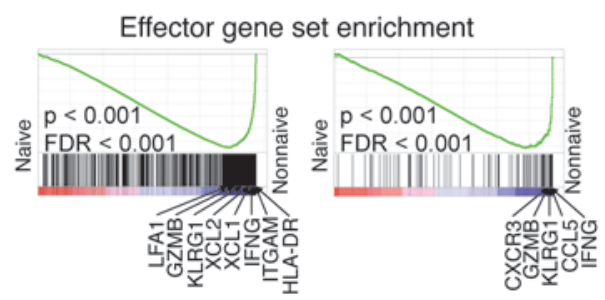

Figure 2

Gene expression of naive and effector T cells from peripheral blood. (A) Two-way hierarchical clustering based on the identified 773 genes, separating all naive from nonnaive $T$ cells. Red indicates overexpression and blue underexpression relative to the mean. Each row represents 1 gene and each column 11,000-cell sample from 1 patient or healthy donor. (B) Relative overexpression of GO terms associated with the identified genes, calculated as described in Methods. (C and D) GSEA of publicly available gene sets describing naive and effector T cells. Positions of selected example genes are indicated. Gene sets comprise genes enriched in naive T cells (C) or in effector cells (D). Genes to the left and right of the rank-ordered list are enriched in naive T cells and nonnaive T cells, respectively.

sets characteristic for the distinction of the 2 populations. From these data, we used 2 gene sets, one which is up- and one which is downregulated in effector $\mathrm{CD}^{+} \mathrm{T}$ cells. Furthermore, in 2007 , Wherry et al. defined gene sets that were up- or downregulated in antigen-specific memory, effector, and exhausted $\mathrm{CD}^{+} \mathrm{T}$ cells from LCMV-infected mice (2). While the gene sets from Willinger et al. described long-term effects of effector differentiation (analysis of total human $\mathrm{CD}^{+} \mathrm{T}$ cell subsets in steady state), the gene sets from Wherry et al. described shorter-term changes of gene expression (model of acute and chronic infection). With Gene Set Enrichment Analysis (GSEA), we determined whether gene sets were enriched in our rank-ordered list of differentially expressed genes. Our naive T cells showed upregulation of the 2 gene sets downregulated in effector cells as identified by Wherry et al. (ref. 2 and Figure 2C) and by Willinger et al. (ref. 31 and Figure 2C). Conversely, the gene sets enriched in our nonnaive $T$ cells were those upregulated in effector cells as defined by Wherry et al. (Figure 2D) and by Willinger et al. (Figure 2D). Together, these data confirm the reproducibility of microarray analysis of highly purified cells, validating our approach of ex vivo analysis of antigen-specific $T$ cells with small cell numbers.

Different gene expression profiles between circulating tumor-and virusspecific T cells. A major aim of our study was to determine whether tumor-specific $\mathrm{CD}^{+} \mathrm{T}$ cells were similar to or different from virusspecific $\mathrm{T}$ cells. By applying the same selection criteria as above (i.e., fold change $\geq 3$, adjusted $P<0.05$ ), we found 390 genes that were differentially expressed between tumor- and EBV-specific $\mathrm{T}$ cells (259 upregulated and 131 downregulated) (Figure 3A and Supplemental Table 2), while only 184 genes (72 upregulated and 112 downregulated) were differentially expressed when compared with CMV-specific T cells (Figure 3B and Supplemental Table 3). Therefore, the differences between CMV-and tumor-specific $\mathrm{T}$ cells were smaller than between EBV- and tumor-specific T cells. A 2-way hierarchical clustering with these probes showed clear distinction between tumor- and EBV-specific (Figure 3C) and tumor- and CMV-specific T cell populations (Figure 3D) from the individual patients and healthy donors despite the high genetic heterogeneity between individuals and the similarity of surface markers of these $\mathrm{T}$ cell populations (Supplemental Figure 3A). Microarray data were confirmed through the analysis of a series of genes by qPCR, among them several inhibitory receptors (Figure 3, E and G). As compared with both EBV-and CMV-specific cells, TIM3 and CTLA4 were upregulated in tumor-specific T cells, while CD160 was upregulated in virus-specific T cells (Figure 3, $\mathrm{E}$ and $\mathrm{G}$ ). $2 \mathrm{~B} 4$ was upregulated in CMV-specific T cells. Interestingly, as compared with EBV-specific T cells, tumor-specific T cells expressed more mRNA encoding granzyme B (GZMB) and granulysin (GNLY), but less XCL1 (lymphotactin). XCL1 was also upregulated in CMV-specific T cells. Finally, we performed a GO term analysis and found that the differences between tumor- and the 2 virus-specific $\mathrm{T}$ cell populations were smaller (Figure 3, $\mathrm{F}$ and $\mathrm{H}$ ) than the differences of naive versus nonnaive $\mathrm{T}$ cells 

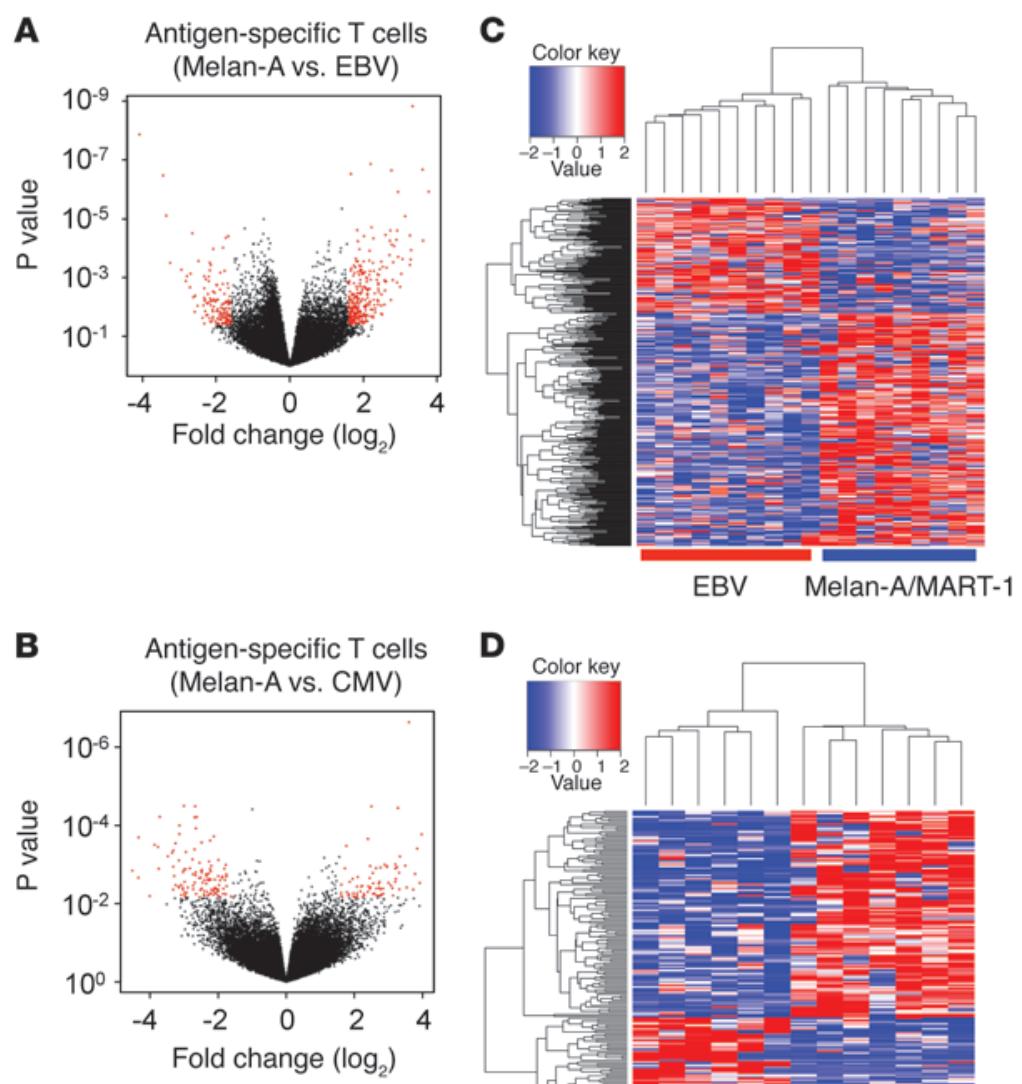

\section{E}

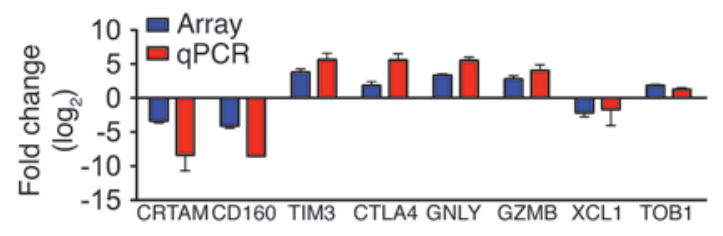

$\mathbf{F}$

Melan-A vs. EBV-specific T cells
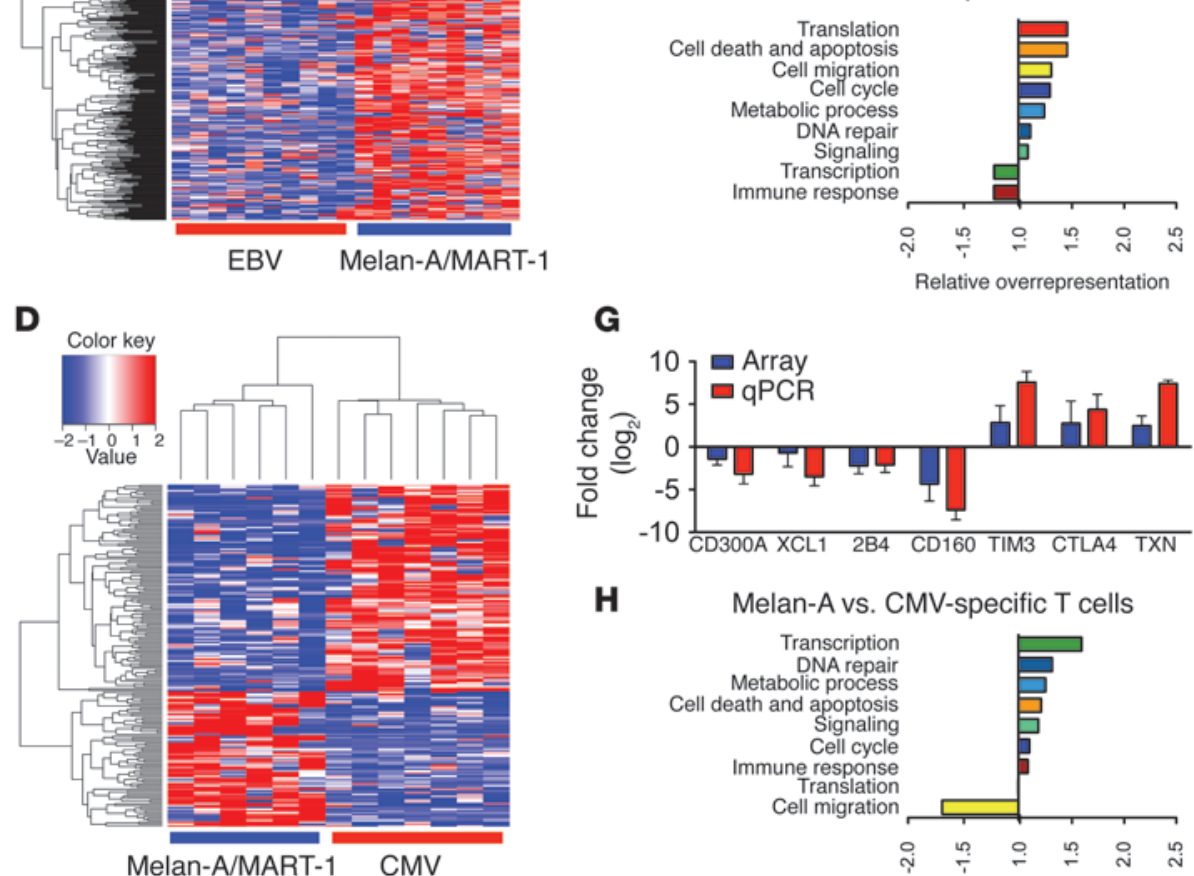

G

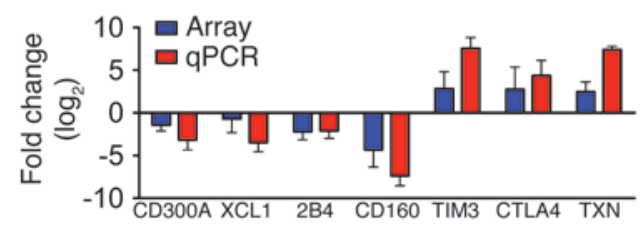

H

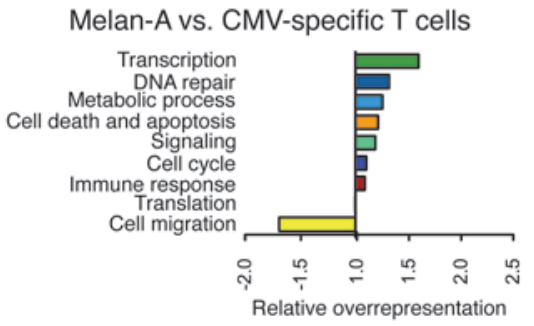

Figure 3

Gene expression of circulating CD8 ${ }^{+} \mathrm{T}$ cells depending on antigen specificity. (A and $\left.\mathbf{B}\right)$ Volcano plots for all gene probes, showing differential expression and $P$ values of the comparison of tumor- versus EBV-specific T cells $(\mathbf{A})$ or tumor- versus CMV-specific T cells (B); diagramming is similar to that in Figure 1. (C and D) Two-way hierarchical clustering based on the identified gene probes separating all tumor-specific $T$ cells from EBV- (C, 405 gene probes corresponding to 390 genes) and from CMV-specific T cells (D, 187 gene probes corresponding to 184 genes). Red indicates overexpression and blue underexpression relative to the mean. Each row represents 1 gene and each column $11,000-c e l l$ sample from 1 patient (tumor-, EBV- and CMV-specific cells) or healthy donor (EBV-specific cells, $n=4)$. (E and G) Log fold changes between tumor- and EBV- (E) or tumor- and CMV-specific T cells $(\mathbf{G})$ of data from microarrays (blue bars) and qPCR (red bars). Positive and negative values indicate overexpression in tumor- and in virus-specific T cells, respectively. Data are represented as mean \pm SEM. (F and $\mathbf{H})$ Relative overexpression of GO terms associated with the identified 390 genes (Melan-A/MART-1 versus EBV; F) or with the identified 184 genes (Melan-A/MART-1 versus $\mathrm{CMV} ; \mathbf{H})$, calculated as described in Methods.

(Figure 2B). Remarkably, immune response genes were not specifically overrepresented relative to a random gene list, suggesting overall similar expression of immune genes in effector $T$ cells specific for EBV, CMV, and Melan-A/MART-1, despite the differences found for inhibitory receptors.

The gene expression profile of circulating tumor-specific $\mathrm{CD}^{+} \mathrm{T}$ cells corresponds to late-differentiated effector cells. EBV- and CMV-specific $\mathrm{T}$ cells are recognized as prototypes of early- and late-differentiated effector cells, respectively (7). This distinction fits with the phenotypes of these 2 populations (Supplemental Figure 3A). We created rank-ordered gene lists to compare tumor-specific with the 2 virus-specific $\mathrm{CD}^{+} \mathrm{T}$ cell populations. The gene sets defined as upregulated in effector cells by Wherry et al. (ref. 2 and Figure 4A) and Willinger et al. (ref. 31 and Figure 4A) were enriched in tumorspecific cells, as compared with their EBV-specific counterparts. In contrast, the only gene set enriched in EBV-specific T cells compared with tumor-specific $T$ cells was the small gene set containing genes specifically upregulated in memory cells when compared with naive $C D 8^{+} \mathrm{T}$ cells as defined by Wherry et al. (Figure 4B). This is likely due to the lower degree of effector differentiation of EBV-specific T cells (which are nevertheless predominantly effector rather than memory cells; Supplemental Figure 3A). When we compared tumor- with CMV-specific $\mathrm{CD}^{+} \mathrm{T}$ cells, we could not find enrichment for any gene set (Figure 4C), confirming the late differentiation stage of tumor-specific T cells. To verify the differential expression of granzyme B and perforin ex vivo on the protein level, we performed intracellular staining. As expected, the tumor- and $\mathrm{CMV}$-specific $\mathrm{CD}^{+} \mathrm{T}$ cells expressed more granzyme $\mathrm{B}$ and perforin than the EBV-specific CD8 ${ }^{+} \mathrm{T}$ cells (Figure 4D). However, all 3 antigen-experienced $T$ cells produced high levels of IFN- $\gamma$ after 4 hours 
A Effector gene set enrichment: Melan-A vs. EBV
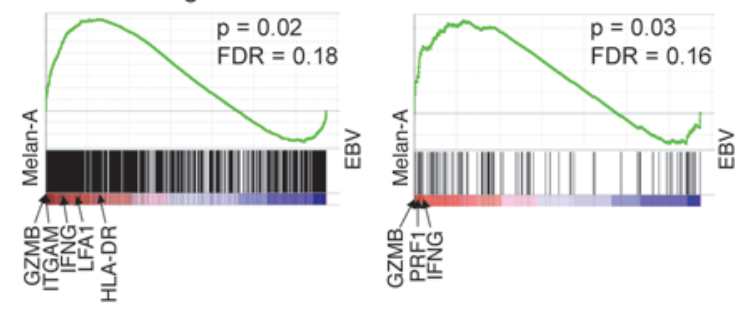

B Memory gene set enrichment in EBV
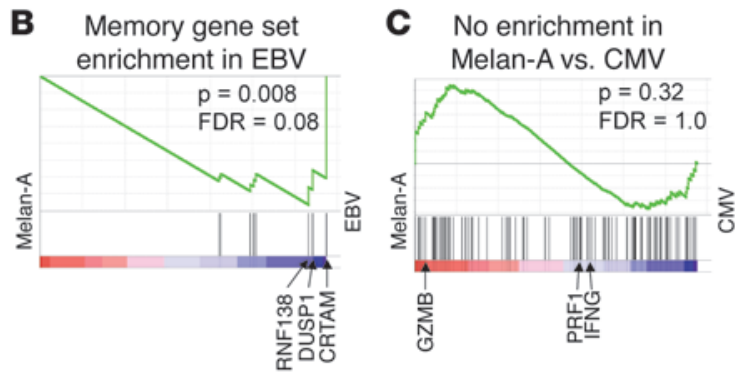

D CMV- and Melan-A-specific cells produce more granzyme B and perforin

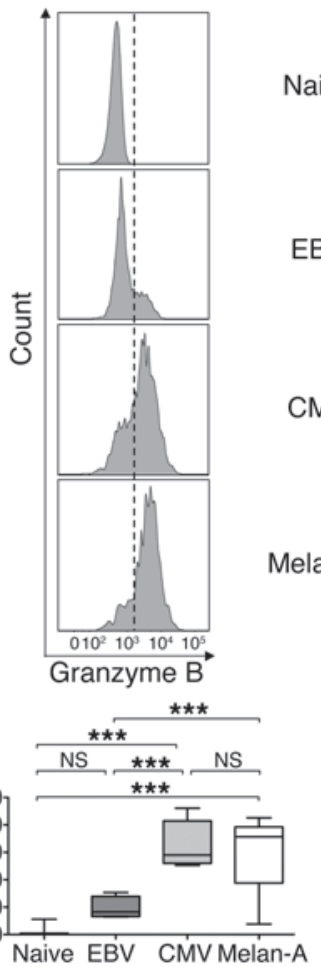

Naive

EBV

CMV
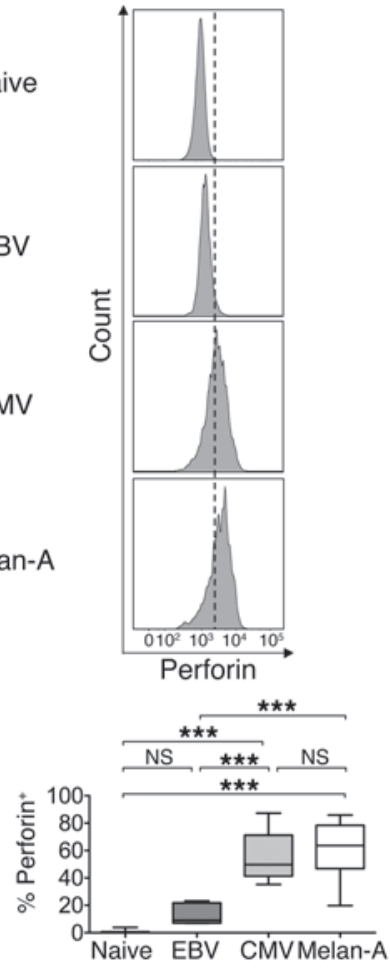

\section{Figure 4}

Circulating tumor-specific T cells are late-differentiated effector cells, resembling CMV-specific T cells. (A) Gene set enrichment of genes describing effector cells (see Figure 2D). Genes to the left and right of the rank-ordered list are enriched in tumor- and EBV-specific T cells, respectively. (B) Gene set enrichment of genes describing memory cells (2). Genes to the left and right of the rank-ordered list are enriched in tumor- and EBV-specific T cells, respectively. (C) No differences were found between Melan-A/MART-1- and CMV-specific T cells, demonstrated by a gene set defining effector cell-related genes (31). (D) Intracellular staining of naive and antigen-specific T cells. Top panels show 1 representative example; below are the combined results of all samples (EBV and CMV, $n=5$; Melan-A, $n=15$; naive, $n=25$ ). Data of EBV-and CMV-specific T cells are from healthy donors, while data of tumor-specific $T$ cells are from patients. ${ }^{* * \star} P<0.001$. Whiskers in box plots indicate maximum and minimum values measured. Line indicates the median.

triggering with peptide-loaded T2 cells (Supplemental Figure 3B). Together, our results demonstrate that tumor- and CMV-specific $\mathrm{CD}^{+} \mathrm{T}$ cells resembled each other closely, while EBV-specific CD8 ${ }^{+}$ $\mathrm{T}$ cells were in earlier stages of effector differentiation.

In contrast to circulating Tcells, tumor-specific Tcells from tumor-infiltrated lymph nodes show an exhaustion profile. Previous studies indicated that functional impairment of tumor-specific $T$ cells may occur primarily in situ $(20,24)$, which was also the case after strong systemic T cell activation by CpG-based vaccination (25). Therefore, we established a clinical investigation protocol to recover large numbers of live cells from tumor-infiltrated lymph nodes (TILN). This enabled us to perform functional studies and gene expression analysis ex vivo from tumor-specific T cells from TILN, in comparison with circulating $\mathrm{T}$ cells. Tumor-specific $T$ cells from metastases showed highly insufficient IFN- $\gamma$ production upon 4-hour peptide triggering (Figure 5A), as published previously $(20,24)$. Microarray analysis allowed the identification of 332 genes (201 up- and 131 downregulated in TILN; Supplemental Table 4) that were differentially expressed between tumor-specific $\mathrm{CD}^{+} \mathrm{T}$ cells from PBMC versus TILN, using the same criteria as before (Figure 5B). Hierarchical clustering using these genes divided the 13 samples into 2 groups only, one for blood and the other for TILN-derived tumor-specific T cells
(Figure 5C). qPCR performed for a selection of genes allowed proper validation (Figure 5D). Among the genes upregulated in tumorspecific cells from TILN were the lymph node retention receptor CRTAM, the chemokines XCL1 and XCL2, the activation marker TNFRSF9, and the inhibitory receptor CTLA4. CXCL13, a B cell chemoattractant usually found in the B cell compartment of lymph nodes, was one of the most highly overexpressed genes. Among the genes downregulated in TILN cells were the cell-growth-regulating protein LYAR and the inhibitory receptor KLRG1. When classifying the differentially expressed genes into broad GO terms, we found that genes involved in cell death and apoptosis and in the immune response were overrepresented compared with a randomly selected gene list (Figure 5E). To obtain a more general overview of the differences of tumor-specific $\mathrm{CD}^{+} \mathrm{T}$ cells from blood versus TILN, we studied gene sets specific for effector cells, naive cells, memory cells, and exhausted cells, as described above. Remarkably, the gene set described for exhausted T cells (2) was significantly enriched in tumor-specific cells from TILN, in contrast with the gene sets characteristic for naive, memory, and effector T cells (Figure 5F). These large-scale data demonstrate an impressive exhaustion profile, with extended molecular alterations of multiple pathways in tumor-specific $\mathrm{CD}^{+} \mathrm{T}$ cells from metastases. 
A

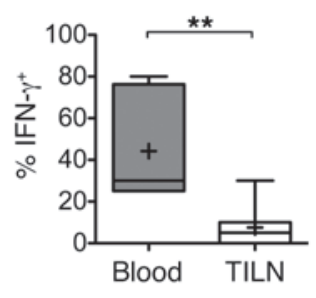

B

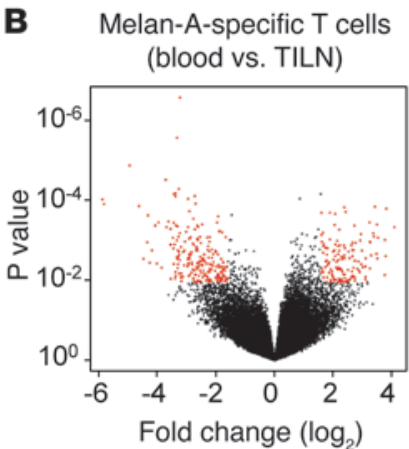

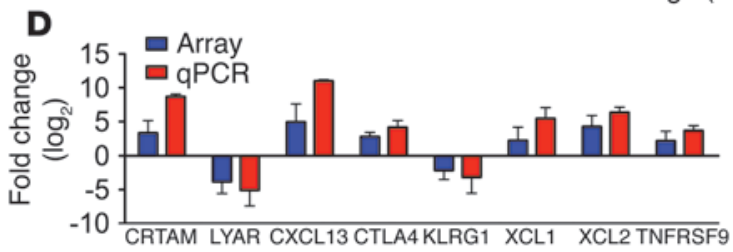
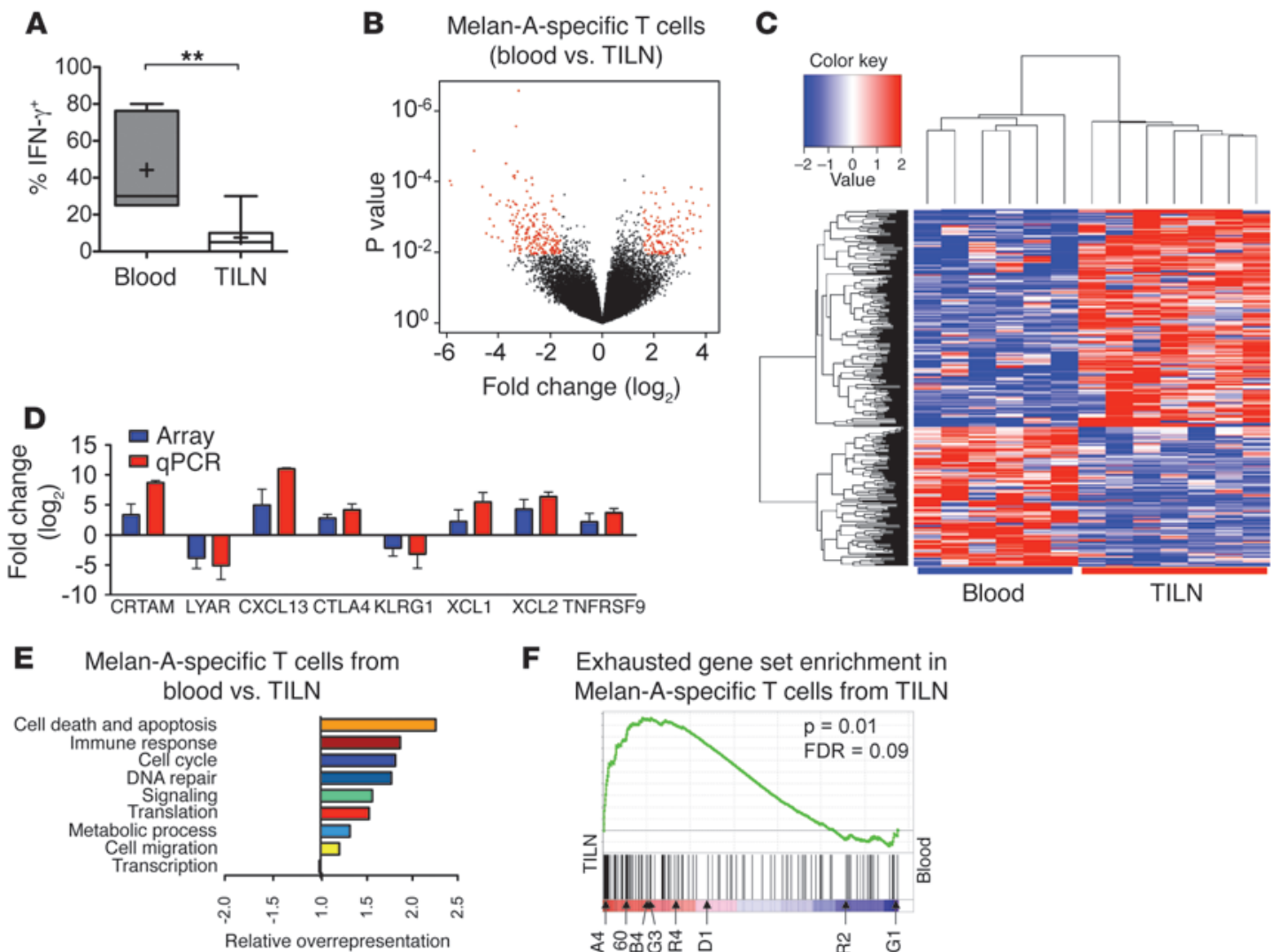

F Exhausted gene set enrichment in Melan-A-specific T cells from TILN

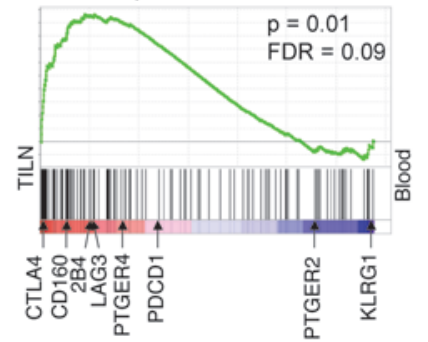

\section{Figure 5}

Exhaustion profile of tumor-specific T cells in situ. (A) IFN- $\gamma$ production by tumor-specific T cells from the circulation (blood; $n=6)$ or TILN ( $n=8$ ) after 4-hour antigen stimulation. Whiskers in box plots indicate maximum and minimum values measured. Cross indicates the mean, while line indicates the median. ${ }^{* *} P<0.01$. (B) Differential gene expression by tumor-specific $\mathrm{T}$ cells isolated from blood versus TILN, as illustrated by a volcano plot for all gene probes. (C) Two-way hierarchical clustering based on the identified 346 genes separating all blood-derived tumorspecific T cells from their TILN counterparts. Red indicates overexpression and blue underexpression relative to the mean. Each row represents 1 gene and each column 1 1,000-cell sample from 1 patient. (D) Log fold change between tumor-specific T cells from blood versus TILN; data from microarrays (blue bars) and qPCR (red bars). Positive and negative values indicate overexpression in tumor-specific T cells from TILN and from blood, respectively. Mean \pm SEM. (E) Relative overexpression of GO terms associated with the identified genes, calculated as described in Methods. (F) Enrichment of the gene set described for exhausted T cells (2) in TILN-derived tumor-specific T cells, relative to their blood-derived counterparts. The positions of inhibitory receptors found in this gene set on the rank-ordered gene list are indicated. A position to the left indicates enrichment in TILN-derived cells, a position to the right enrichment in blood-derived cells.

Enhanced expression of inhibitory receptors, such as CTLA4 and LAG3, was observed in $\mathrm{T}$ cell exhaustion (2, 23, 32-34). Interestingly, their expression was enriched in TILN cells, with the notable exceptions of PTGER2 and KLRG1 (Figure 5F). However, KLRG1 was described as more strongly expressed in functionally competent effector cells than in exhausted T cells (2), compatible with our data. The absolute expression values of selected inhibitory receptors are detailed in Table 1. Although it seems likely that the tumor microenvironment plays a role, the reasons for the observed enhanced expression of inhibitory receptors remain to be elucidated.

Differential protein expression of multiple inhibitory receptors by tumorand virus-specific $C D 8^{+} T$ cells. To determine expression of inhibitory receptors at the protein level, we produced tetramers labeled with (multiple) different fluorochromes and used them in combination with several monoclonal antibodies (multi-tetramer staining; Figure 6A). Compatible with mRNA data, CD160 and 2B4 were more frequently expressed by both EBV-and CMV-specific T cells than by tumor-specific T cells from peripheral blood (Figure 6B), in agreement with a study reporting that most $\mathrm{CD} 160^{+}$cells coexpressed 2B4 (35). In contrast, circulating tumor-specific $T$ cells expressed more TIM- 3 and more PD- 1 than the 2 virus-specific T cell populations (Figure 6B), in line with 2 recent reports of TIM- $3^{+} \mathrm{PD}-1^{+}$ cells among tumor-specific T cells $(23,36)$. Large percentages of PD- $1^{+}$tumor-specific T cells coexpressed TIM-3 and/or KLRG-1. Similar results were obtained when we analyzed the mean fluorescence intensity (Supplemental Figure 4). Our technique allowed analyzing simultaneous coexpression of multiple inhibitory receptors, for CD160, KLRG-1, PD-1, and TIM-3, or for 2B4, LAG-3, and CTLA-4. We found a pronounced increase in inhibitory receptor coexpression from naive to central memory, effector memory, and effector memory $\mathrm{RA}^{+}$cells (data not shown). On antigen-specific $\mathrm{T}$ cells, there were various combinations of inhibitory receptors. Melan-A-specific T cells from TILN expressed more CTLA-4, LAG-3, and TIM-3, but less KRLG-1 than their counterparts from 


\section{Table 1}

Expression of selected inhibitory receptors by tumor-specific T cells

\begin{tabular}{lcccc} 
& \multicolumn{2}{c}{ From TILN } & \multicolumn{2}{c}{ From blood } \\
& Mean & SD & Mean & SD \\
CTLA4 & 12.1 & 0.6 & 9.3 & 2.6 \\
CD160 & 8.6 & 2.2 & 7.6 & 2.0 \\
LAG3 & 11.5 & 1.2 & 10.8 & 0.9 \\
2B4 & 8.3 & 1.2 & 7.7 & 1.0 \\
PTGER4 & 9.5 & 1.8 & 9.0 & 3.1 \\
KLRG1 & 6.5 & 1.3 & 8.7 & 1.7
\end{tabular}

Absolute expression values and SD of selected inhibitory receptors on a $\log _{2}$ scale.

peripheral blood (Figure 6C), confirming the results obtained by the microarray analysis. These data reveal a high level of heterogeneity, with multiple antigen-specific T cell subpopulations expressing different combinations of inhibitory receptors. It is likely that many of these subpopulations are effector memory cells and effector memory $\mathrm{RA}^{+}$, as they make up the vast majority of Melan-Aspecific T cells (Supplemental Figure 3A). Naive and central memory cells were infrequent, but may nevertheless contribute to this heterogeneity. Furthermore, extended studies are necessary to determine the functional impact of coexpressed inhibitory receptors. Finally, the marked differences between tumor-, CMV-, and EBV-specific T cells suggest different roles of inhibitory receptors in viral infection versus cancer.

\section{Discussion}

In peripheral blood, tumor-specific $\mathrm{T}$ cells induced by vaccination showed an effector cell profile (Supplemental Figure 5), similar to CMV-specific T cells and similar to the murine counterpart of $\mathrm{CD}^{+} \mathrm{T}$ cells in acute LCMV Armstrong infection (2). Differentiation of EBV-specific $\mathrm{CD}^{+} \mathrm{T}$ cells was less pronounced, but they nevertheless resembled effector cells. In contrast to these 4 effector cell populations, tumor-specific $T$ cells in situ displayed an exhaustion profile, with significant similarity to murine $\mathrm{T}$ cells in chronic infection with LCMV clone 13 (2).

Tumor-infiltrating $\mathrm{T}$ cells are functionally deficient $(20,23$, $24,34)$, which is likely coresponsible for the limited efficacy of immunotherapy. However, the underlying mechanisms remain poorly characterized, in contrast with chronic infectious diseases $(1,2)$. Our finding of $\mathrm{T}$ cell exhaustion in melanoma metastases results from what we believe is the first comprehensive molecular characterization of self- and tumor-specific $\mathrm{T}$ cells, providing explanations for their functional impairment. Tumor-specific $\mathrm{T}$ cells from metastases showed considerable molecular alterations, with surprisingly strong overexpression of many genes regulating various cell functions. This included genes involved in immune responses, cell death and apoptosis, and cell cycle and DNA repair. Thus, the data point to enhanced immune activation and apoptosis, and problems in maintaining DNA integrity and sustaining cell cycling in $\mathrm{T}$ cells of metastases.

We did not find significant correlations between our $T$ cell data and clinical results (e.g., patient survival). However, phase I studies such as the present trial of immunotherapy are not suited for clinical outcome analysis. Rather, they are designed for providing enhanced biological insight. Indeed, we identified specific molecular alterations potentially representing molecular targets for improved therapy. Nevertheless, further studies are required to determine which of these targets are most promising for evaluation in large-scale phase III clinical trials.

Based on the available evidence for functional $\mathrm{T}$ cell impairment in HIV-1, HBV, and HCV infections (11, 16-18, 37), it will be useful to perform comparative molecular profiling of $\mathrm{T}$ cells in different infections and malignancies in order to identify similarities and differences, providing the rational basis for therapy optimization. Very recently, HIV-1-specific T cells have been profiled, with identification of $\mathrm{T}$ cell exhaustion and BATF upregulation by PD-1 in patients failing to control HIV infection (18). Even though we did not find enhanced BATF expression in tumor-specific $T$ cells from TILN, we observed similarities in gene expression signatures and upregulation of multiple inhibitory receptors on tumor-specific $\mathrm{T}$ cells also at the protein level.

Besides analysis of tumor-specific $\mathrm{T}$ cells after vaccination, it would be interesting to profile spontaneously arising $\mathrm{T}$ cell responses and naive tumor-specific $T$ cells from patients and healthy donors, with the aim of identifying disease mechanisms responsible for altered $\mathrm{T}$ cell function. We expect that tumor-specific $T$ cells from healthy donors would show an expression profile similar to total naive $\mathrm{CD} 8^{+} \mathrm{T}$ cells, while spontaneously responding $\mathrm{T}$ cells may show some degree of effector cell differentiation. However, such studies are technically challenging, since tumor-specific $\mathrm{T}$ cells in healthy donors and early stages of cancer are rare and difficult to isolate for ex vivo analysis. Therefore, laboratory techniques must be optimized for comprehensive characterization of even smaller cell numbers, ultimately down to the single cell level.

$\mathrm{T}$ cell tolerance to self and tumor antigens is assured by negative selection in the thymus and through anergy induction and $T$ cell deletion in the periphery. Anergy has been characterized in at least 9 different experimental settings, most of them in vitro models and/or CD4+ $\mathrm{T}$ cell models (26). Unfortunately, no comprehensive gene expression data are available. Therefore we could not systematically evaluate anergy in our study. Nevertheless, we made an attempt by evaluating 29 anergy-related genes described in a model of ionomycin-induced anergy and a model of deletional tolerance $(38,39)$. We found that some of these genes (e.g., $C B L B$ and CTLA4) were enriched in tumor-specific T cells from metastases (Supplemental Table 4), but most of the described genes (e.g., $I T C H, E G R 2$, and DGKZ) were not enriched (not shown).

Our study was performed in patients with advanced stage III-IV melanoma. It has been hypothesized that late cancer stages may be associated with $\mathrm{T}$ cell exhaustion (1), whereas anergy and tolerance would be induced already at early stages of tumorigenesis $(40,41)$. Possibly, self- and tumor-specific T cells may show discrete alterations already at the naive stage and/or after spontaneous activation. Perhaps anergy mechanisms are functional even at later disease stages. The elucidation of these points requires further methodological progress. For the time being, our data support the conclusion that exhaustion likely contributes to the functional deficiencies, but does not rule out the involvement of further mechanisms such as anergy or self tolerance.

In circulating tumor-specific $\mathrm{T}$ cells, we found effector cell signatures compatible with their ample production of granzyme B and perforin (Figure 4) and efficient expression of IFN- $\gamma$ upon 4-hour triggering with antigen (Supplemental Figure 3B and refs. $20,21)$. Due to the high efficacy of CPG 7909 as adjuvant, the circulating tumor-specific $\mathrm{T}$ cells studied here were more strongly 
A

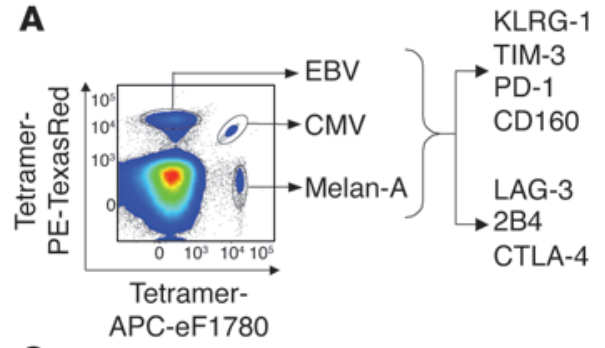

C

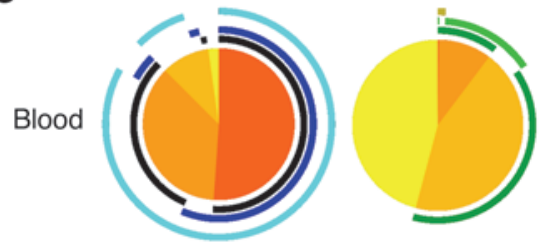

TILN

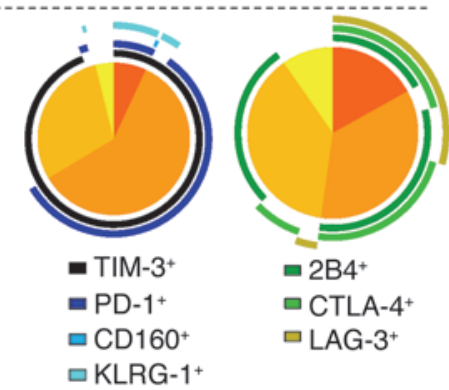

Number of inhibitory receptors

$=4 \quad 3 \quad \square 2 \square 1 \square 0$

B

CM

EBV

CMV
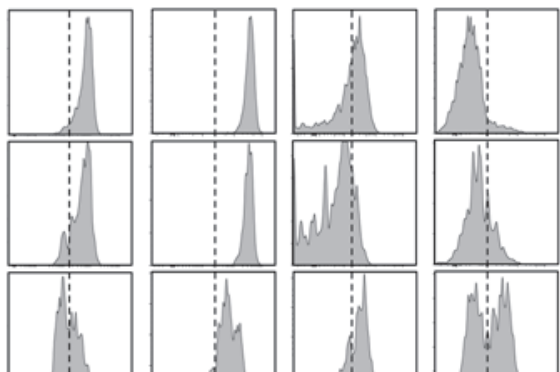

$\sum_{\substack{\frac{\pi}{2} \\ \frac{\pi}{0}}}^{\text {Blood }}$

TILN
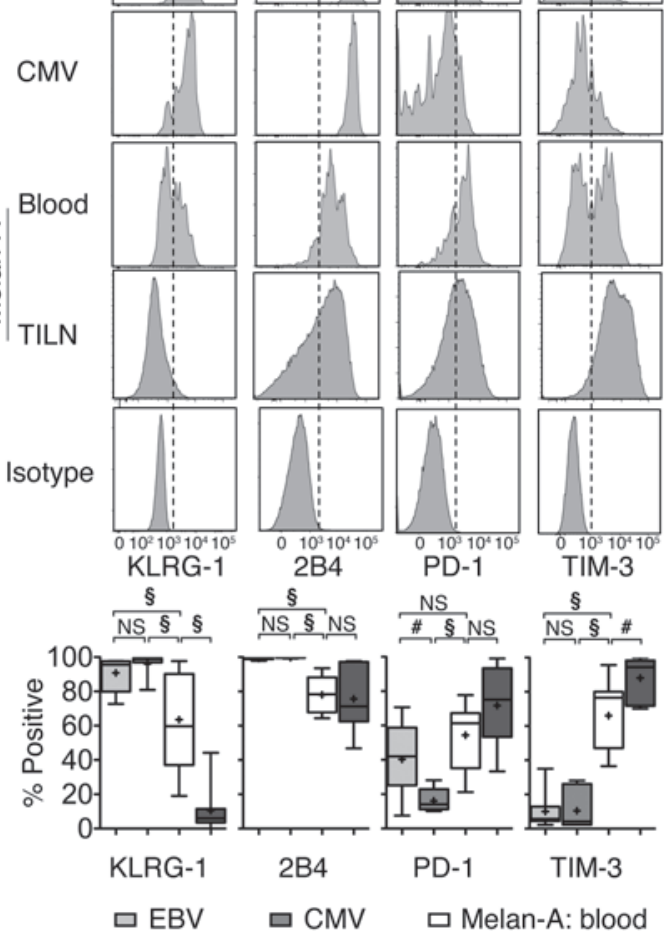

TIM-3

§ $P D-1$
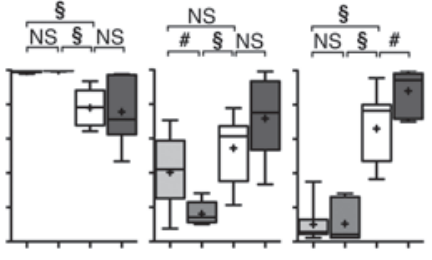

2B4

PD-1

TIM-3
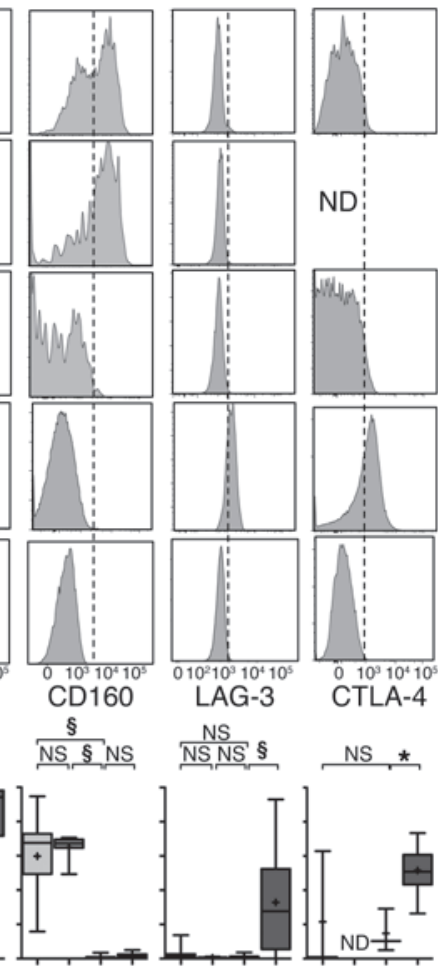

CD160

LAG-3

- Melan-A: TILN

Figure 6

Multi-tetramer staining assessing coexpression of inhibitory receptors. (A) Staining with tetramers binding to EBV- (PE-Texas Red), Melan-A/ MART-1- (APC-eFluor 780), or CMV- (PE-Texas Red and APC-eFluor 780) specific T cells (labeling tetramers with 2 instead of 1 fluorochrome identifies larger numbers of epitope-specific $T$ cell populations than the number of fluorescence channels used). T cells were analyzed for coexpression of 7 inhibitory receptors: KLRG-1 (Alexa Fluor 488), TIM-3 (PE), PD-1 (PerCP-eFluor710), and CD160 (Alexa Fluor 647), or LAG-3 (FITC), 2B4 (PE-Cy5.5), and CTLA-4 (APC). (B) Expression of 7 different inhibitory receptors. Histograms of a representative sample are gated on $\mathrm{CD}^{+}$tetramer ${ }^{+}$cells. Box plots summarize the data of all patients analyzed (EBV, $n=16$; CMV, $n=6$; Melan-A blood, $n=10$, except for CTLA-4, $n=3$; Melan-A TILN, $n=8-9$ ). Whiskers in box plots indicate the maximum and minimum values measured. Cross indicates the mean, while line indicates the median. ${ }^{\star} P<0.05$; $\# P<0.01 ; \S P<0.001$. (C) Coexpression of 0 to 4 and 0 to 3 inhibitory receptors was analyzed with SPICE (48).

activated (19) than in most other cancer vaccine studies with their lower frequencies and less pronounced effector cell differentiation. Thus, our data of circulating cells are not representative for the latter, but nevertheless demonstrate that self- and tumor-specific $T$ cells have the potential to become effector cells. Despite the high efficacy of the adjuvant used, we could not observe significant bystander effects on circulating $\mathrm{T}$ cells with specificities other than for the vaccine (Figure 1).

One could argue that vaccination should have activated the tumor-specific T cells to an even higher degree than CMV-specific $\mathrm{T}$ cells in healthy donors (which was actually the case in some of our melanoma patients; our unpublished observations). Protection from latent CMV is likely less demanding for $\mathrm{T}$ cells than protection from acute viral disease. Possibly, even more strongly activated $T$ cells may be required for protection from cancer progression. Indeed, adoptive transfer therapy has shown that tumorspecific $T$ cells at much higher frequency and strong activation can eliminate large melanoma metastases (22). Molecular profiling of these cells in comparison with $\mathrm{T}$ cells during acute viral infections may reveal eventual differences from our data. Alternatively, therapeutic success and protection from disease may be primarily achieved due to high numbers of $\mathrm{T}$ cells with molecular properties similar to those described here. However, patients with acute viral infections are rarely accessible for clinicians and researchers. Moreover, antitumor vaccines rarely induce $T$ cell responses comparable to acute viral infections. In contrast to vaccines consisting of synthetic molecules and inactivated pathogens, live vaccines (essentially vaccinia virus and yellow fever vaccine) can induce high $\mathrm{T}$ cell frequencies $(42,43)$ and efficient protection. Comprehensive profiling of these $\mathrm{T}$ cells is feasible and may likely contribute to identifying protective mechanisms of human T cells.

Differentiation from naive to effector $\mathrm{T}$ cells introduces large changes in expression of not only immune response genes, but also of genes involved in translation, in cell death and apoptosis, and in cell migration. These changes result in increased production of effector molecules, migration to pathologic tissue, and cell survival. Based on GO terms, we compared EBV-with circulating tumorspecific $T$ cells and found that the latter overexpressed genes involved in translation, cell death, and apoptosis, likely reflecting the fact that the tumor-specific T cells were more advanced in 
effector cell differentiation. Compared with CMV-specific T cells, circulating tumor-specific $\mathrm{T}$ cells expressed slightly more genes related to transcription, but fewer genes involved in cell migration. Despite these distinctions, the 3 effector cell populations from peripheral blood were relatively similar.

Inhibitory receptors were prominent among the differentially expressed genes. This group of genes is attracting increasing attention, also because of its importance in $\mathrm{T}$ cell exhaustion and therapeutic potential (2). Nevertheless, the circulating tumor-specific $\mathrm{T}$ cells expressed granzyme B and perforin at high levels and were functionally competent (19). Apparently, effector cells can express inhibitory receptors but nevertheless maintain functional competence. Our study demonstrates coexistence of functional cells in circulation and exhausted cells in metastases. We have preliminary data indicating that this may occur even within individual $\mathrm{T}$ cell clonotypes (our unpublished observations). It appears that migration of T cells into the tumor tissue is associated with downregulation of cytokine production and exhaustion as a consequence of encountering inhibitory receptor ligands expressed in the tumor tissue, in conjunction with antigen recognition. Thus, exhaustion of $\mathrm{T}$ cells in metastases but not in peripheral blood may be linked to the frequent and strong expression of these ligands in the tumor microenvironment. This interpretation is compatible with our earlier findings that the functional deficiency of tumor-residing $T$ cells is readily reversible, since $T$ cells from metastases regain function after 1 to 2 days culture in vitro $(20,44)$.

$\mathrm{HCV}$-specific T cells may coexpress up to 4 of the inhibitory receptors KLRG1, 2B4, CD160, and PD-1, correlating with CD127 downregulation and functional impairment (32). Even though many tumor-specific $T$ cells expressed KLRG1, 2B4, PD-1, and TIM-3, they did not express CD160. This difference may be functionally relevant for HCV-versus tumor-specific T cells. Moreover, expression of inhibitory receptors was more abundant in $\mathrm{T}$ cells from TILN as opposed to blood. The differential coexpression of multiple inhibitory receptors in viral infection versus cancer, and depending on antigen specificity/differentiation status and anatomical localization, suggests that the functional regulation of antigen-specific T cells is more complex than previously thought.

In summary, our study provides comprehensive molecular profiles of human $\mathrm{CD}^{+} \mathrm{T}$ cells. Although tumor-specific $\mathrm{T}$ cells can acquire substantial effector cell properties, they display an exhaustion profile in metastases. With modern technologies applied to small cell numbers, it becomes increasingly possible to determine whether functional impairment and molecular exhaustion of tumor-specific T cells are due to their specificity for self antigen, and/or immune suppression in situ.

\section{Methods}

Healthy donors, melanoma patients, lymphocyte isolation, and flow cytometry. Blood from $4 \mathrm{~A}^{+}$healthy donors was obtained from the university blood transfusion center of Lausanne, Switzerland. Peripheral blood and surgery specimens were obtained from $\mathrm{A}^{*} 0201^{+}$patients with stage III/IV metastatic melanoma. Patients had received multiple monthly low-dose vaccinations s.c. with $100 \mu \mathrm{g}$ Melan-A/MART-1 peptide and CpG (500 $\mu \mathrm{g}$ PF-3512676/7909; provided by Pfizer/Coley Pharmaceutical Group), emulsified in IFA (300-600 $\mu$ l Montanide ISA-51; provided by Seppic) as described previously (19). Analysis of circulating tumor-specific T cells was done after $11 \pm 5$ monthly vaccinations; the last was at a mean of 96 days before blood withdrawal. Tumor-specific T cells from TILN were prepared after finely mincing surgery specimens, which were obtained after $7 \pm 2$ monthly vaccinations, the last at a mean of 79 days before surgery. Vaccinations were done in the context of Ludwig Institute for Cancer Research trials $(19,45)$ and approved by the Ludwig Institute for Cancer Research protocol review committee as well as by the medical and ethical committees of the University Hospital (Lausanne). Blood and tissue were obtained upon informed patient consent, and the study was performed according to the relevant regulatory standards. Mononuclear cells were purified by density gradient using Lymphoprep (Axis-Shield) and immediately cryopreserved in RPMI 1640 supplemented with 40\% FCS and 10\% DMSO.

For microarray analysis, 1,000 cells from each sample were sorted using a Vantage SE directly into lysis and storage buffer provided by Miltenyi Biotec as shown in Supplemental Figure 1. $\mathrm{CD}^{+} \mathrm{T}$ cells were enriched using magnetic bead sorting (Miltenyi Biotec). Cells were stained on ice and diluted at one million cells/ml. Cells were stained with CD8-specific antibody, the dead cell marker DAPI, and either with lineage markers (CD4, CD14, CD16, CD19) together with A2/EBV BMLF1 $280-288$ (GLCTLVAML), A2/ CMV pp65 495-503 (NLVPMVATV), or A2/Melan-A/MART-1 26-35A27L (ELAGIGILTV) tetramers binding to high- and low-affinity $\mathrm{T}$ cell receptors (46) or with CD45RA-, CCR7-, CD28-, and CD27-specific antibodies. Naive $\mathrm{T}$ cells were defined as $\mathrm{CD} 8^{+} \mathrm{CD} 45 \mathrm{RA}{ }^{+} \mathrm{CCR} 7^{+} \mathrm{CD} 27^{+} \mathrm{CD} 28^{+}$. The sorting strategy is shown in Supplemental Figure 1. Manipulations were done at $4^{\circ} \mathrm{C}$, avoiding gene expression alteration due to staining and sorting. Sorting purity was high, as determined by analyzing aliquots before and after FACS sorting. Representative examples are shown in Supplemental Figure 1, B-D. Among CD8 ${ }^{+} \mathrm{T}$ cells, percentages for A2/EBV tetramer ${ }^{+}$ cells were $1.00 \pm 0.89$ ( 4 healthy donors and 12 patients); for A2/CMV tetramer ${ }^{+}$cells, $1.53 \pm 1.08$ (7 patients); and for A2/Melan-A/MART-1 tetramer+ cells, $1.43 \pm 1.31$ in blood (11 patients) and $3.35 \pm 3.35$ in TILN (7 patients). After sorting, lysed cells were incubated for 10 minutes at $45^{\circ} \mathrm{C}$ and then directly frozen at $-80^{\circ} \mathrm{C}$.

Intracellular antibody staining was performed as previously described (27). In brief, cells from the $\mathrm{CD}^{+}$fraction were first stained with PE-labeled tetramers, followed by anti-CD8-Pacific Blue antibody. After washing in PBS, cells were incubated with LIVE/DEAD-Fixable-Aqua (Invitrogen) for dead cell exclusion, and fixed at room temperature (RT) during 30 minutes ( $1 \%$ formaldehyde buffer). Cells were washed and stained with $\mathrm{mAbs}$ anti-perforin-FITC or anti-granzyme B-FITC (BD) in FACS buffer with $0.1 \%$ saponin for 30 minutes at $4^{\circ} \mathrm{C}$. For the staining of IFN- $\gamma, \mathrm{CD} 8^{+}$cells were stimulated with peptide-loaded $\mathrm{T} 2$ cells for 4 hours in the presence of Brefeldin-A (Sigma-Aldrich) prior to antibody staining with anti-IFN- $\gamma-$ PE-Cy7 (BD Pharmingen). Data of IFN- $\gamma$-production from tumor-specific $\mathrm{T}$ cells were previously published (20).

For antibody staining of multiple inhibitory receptors, samples were purified and enriched as described above and then stained using tetramers detecting the same EBV, CMV, or Melan-A/MART-1 epitopes as described above. Melan-A-specific tetramers were labeled with APC-eFluor 780 (eBioscience), EBV-specific tetramers were labeled with PE-Texas Red (BD Pharmingen), and CMV-specific tetramers were labeled with both APC-eFluor 780 and PE-Texas Red, allowing for individual analysis of T cells specific for the 3 epitopes in a single sample (multi-tetramer staining technique; ref. 47). After 45 minutes at $4^{\circ} \mathrm{C}$, cells were washed and surface staining was performed for CD8, CCR7, CD45RA and (a) LAG-3 (Alexis Biochemicals) and 2B4 (BioLegend) or (b) KLRG-1 (gift from H.-P. Pircher, Department of Immunology, University of Freiburg, Freiburg, Germany), TIM-3 (R\&D Systems), PD-1 (eBioscience), and CD160 (eBioscience). Samples (a) were fixed at room temperature for 30 minutes ( $1 \%$ formaldehyde buffer) and then stained for CTLA-4 (BD Biosciences - Pharmingen) in FACS buffer with $0.1 \%$ saponin for 30 minutes at $4^{\circ} \mathrm{C}$. LIVE/DEADFixable-Aqua (Invitrogen) was used as a dead cell exclusion marker, and appropriate isotype controls were used to define negative populations. 
Data were acquired on a Gallios Flow Cytometer (Beckman Coulter) and analyzed using FlowJo 9.1 (TreeStar). Analysis of coexpression of inhibitory receptors used SPICE version 5.1 (48).

Microarray and $q P C R$. Gene expression profiling was done in 2 experiments. The first experiment included samples from blood-derived naive, EBV-, and tumor-specific T cells. The second experiment included tumorspecific $\mathrm{T}$ cells from blood and metastasis, and CMV-specific T cells from blood. Frozen samples were sent to Miltenyi Biotec and processed according to the vendor-recommended protocol for gene expression analysis. Samples were hybridized to Agilent Whole Human Genome Oligo Microarrays $4 \times 44 \mathrm{~K}$ and scanned using the Agilent microarray scanner system (Agilent). The Agilent Feature Extraction Software was used for readout and processing of image files. Background correction, filtering of data, and quantile normalization were done using the Agi4 $\mathrm{x} 44$ PreProcess software package as described in the package manual. The Limma software package was used to identify the differentially expressed genes and creation of rank-ordered lists. We analyzed eventual contaminations from B cells, monocytes, and dendritic cells, and found that expression levels of IGHG1, CD19, TLRs, and $\mathrm{CD} 1$ were between $0.28 \%$ and $2.72 \%$ of the respective expression of $\mathrm{CD} 3 \mathrm{E}$, confirming the high purity of our samples. We also evaluated intragroup variability possibly leading to high background. For this, we randomly split the data from 13 naive $\mathrm{CD}^{+} \mathrm{T}$ cell samples into 3 pairs of 2 groups of 6 and 7 samples each and analyzed differences between the groups. We found that none of the gene probes were different in any of the pairings, demonstrating that the background was low (data not shown). For nonnaive cells (Figure 2), the data from EBV- and tumor-specific CD8 ${ }^{+}$ $\mathrm{T}$ cells were pooled. Genes were assigned to broad GO terms using the GO Term Mapper (http://go.princeton.edu/cgi-bin/GOTermMapper), yielding both the percentage of submitted genes attributed to a given GO term versus the percentage of all annotated genes attributed to that GO term. Relative overrepresentation was calculated by dividing the percentage of submitted genes attributed to a GO term by the percentage of all available genes annotated with this GO term. Rank-ordered gene lists (ranked according to the B value) were analyzed with GSEA (www.broadinstitute. org/gsea; ref. 49). Enrichment was considered significant if $P$ was less than 0.05 and FDR was less than 0.25 as suggested in the online tool.

qPCR was performed to validate the enriched genes observed in microarray experiments. Custom-ordered oligos (Microsynth) were designed using the online tool from Universal Roche Library Assay Design Centre (Supplemental Table 5). Reaction mix used was Power Sybr Green Master Mix (Applied Biosystems), and amplification was monitored with Applied Biosystems 7900HT Fast Real-Time PCR System (15-minute enzyme activation and
40 cycles of 15 seconds $95^{\circ} \mathrm{C}, 1$ minute $60^{\circ} \mathrm{C}$ ). A Hamilton Liquid Handling Robotic System was used to assemble the 384-well plates. Amplified cDNA samples used for microarray analysis were diluted (1:50) and used for qPCR after confirming the linear and single product amplification by the primers. Samples were measured in triplicate. GAPDH was used as a housekeeping gene to calculate relative expression values.

Statistics. For quantitative comparisons, Student's $t$ test (2-sample 2-tailed comparison) or 1-way ANOVA with Tukey post-test (multiple-sample comparison) was performed with Prism 5.0; $P<0.05$ was considered as significant. $P$ values and FDRs for GSEA were calculated with 1,000 permutations in the online tool. Microarray analysis was done with relatively restrictive criteria, i.e., by considering gene probes as significant if the $P$ value, corrected for a FDR of 0.05 , was $P \leq 0.05$ and the fold change was $\geq 3$.

Accession numbers. The gene-expression data described in this paper have been deposited in the NCBI Gene Expression Omnibus and are accessible through the GEO accession number GSE24536.

\section{Acknowledgments}

We are obliged to the patients for their dedicated collaboration. We gratefully acknowledge M. Delorenzi, F. Schütz, H.-P. Pircher, M. Etzrodt, M. Pittet, M. Matter, O. Michielin, L.J. Old, J. O’DonnellTormey, E.W. Hoffman, and A. Krieg for essential contributions; D. Zehn, P. Ohashi, H.R. MacDonald, J. Skipper, and H.F. Oettgen for support; and P. Schneider, L. Derre, M. Braun, C. Christiansen-Jucht, C. Jandus, J.-P. Rivals, T. Lövgren, and M. Iancu for collaboration and advice. We thank P. Guillaume and I. Luescher for tetramers, and Pfizer and Coley Pharmaceutical Group (USA) for providing CPG 7909 (PF-3512676). This work was supported by the Ludwig Institute for Cancer Research, the Cancer Research Institute (USA), the Cancer Vaccine Collaborative, Atlantic Philanthropies (USA), the Wilhelm Sander-Foundation (Germany), the Swiss Cancer League (grant 02279-08-2008), the Swiss National Science Foundation, and the Swiss National Center of Competence in Research (NCCR) Molecular Oncology.

Received for publication December 14, 2010, and accepted in revised form March 16, 2011.

Address correspondence to: Daniel Speiser, Ludwig Institute for Cancer Research, Hôpital Orthopédique, 05/1552, Av. P.-Decker 4, CH-1011 Lausanne, Switzerland. Phone: 41.21.314.0182; Fax: 41.21.314.7477; E-mail: d.e.speiser@gmail.com.
1. Kim PS, Ahmed R. Features of responding T cells in cancer and chronic infection. Curr Opin Immunol. 2010;22(2):223-230.

2. Wherry EJ, et al. Molecular signature of CD8+ $\mathrm{T}$ cell exhaustion during chronic viral infection. Immunity. 2007;27(4):670-684.

3. Turner SJ, Kedzierska K, La Gruta NL, Webby R, Doherty PC. Characterization of CD8+ T cell repertoire diversity and persistence in the influenza A virus model of localized, transient infection. Semin Immunol. 2004;16(3):179-184.

4. Gallimore A, Hengartner H, Zinkernagel R. Hierarchies of antigen-specific cytotoxic $\mathrm{T}$ cell responses. Immunol Rev. 1998;164:29-36.

5. Yewdell JW, Bennink JR. Immunodominance in major histocompatibility complex class I-restricted T lymphocyte responses. Annu Rev Immunol. 1999; 17:51-88.

6. Appay V, Douek DC, Price DA. CD8+ T cell efficacy in vaccination and disease. Nat Med. 2008; 14(6):623-628.

7. Appay V, Rowland-Jones SL. Lessons from the study of $\mathrm{T}$ cell differentiation in persistent human virus infection. Semin Immunol. 2004;16(3):205-212.

8. Makedonas G, et al. Perforin and IL-2 upregulation define qualitative differences among highly functional virus-specific human $\mathrm{CD}^{+} \mathrm{T}$ cells. PLoS Pathog. 2010;6(3):e1000798.

9. Chen SF, et al. Antiviral CD8 ${ }^{+} \mathrm{T}$ cells in the control of primary human cytomegalovirus infection in early childhood. J Infect Dis. 2004;189(9):1619-1627.

10. Guerreiro M, et al. Human peripheral blood and bone marrow Epstein-Barr virus-specific T cell repertoire in latent infection reveals distinct memory $\mathrm{T}$ cell subsets. Eur J Immunol. 2010;40(6):1566-1576.

11. Zajac AJ, et al. Viral immune evasion due to persistence of activated T cells without effector function. J Exp Med. 1998;188(12):2205-2213.

12. Moskophidis D, Lechner F, Pircher H, Zinkernagel $\mathrm{RM}$. Virus persistence in acutely infected immunocompetent mice by exhaustion of antiviral cytotoxic effector T cells. Nature. 1993;362(6422):758-761.

13. Wherry EJ, Blattman JN, Murali-Krishna K, van der Most R, Ahmed R. Viral persistence alters CD8 ${ }^{+} \mathrm{T}$ cell immunodominance and tissue distribution and results in distinct stages of functional impairment. J Virol. 2003;77(8):4911-4927.

14. Yi JS, Cox MA, Zajac AJ. T cell exhaustion: characteristics, causes and conversion. Immunology. 2010; 129(4):474-481.

15. Barber DL, et al. Restoring function in exhausted $\mathrm{CD}^{+} \mathrm{T}$ cells during chronic viral infection. Nature. 2006;439(7077):682-687.

16. Rehermann B, Nascimbeni M. Immunology of hepatitis B virus and hepatitis $C$ virus infection. Nat Rev Immunol. 2005;5(3):215-229.

17. Letvin NL, Walker BD. Immunopathogenesis and immunotherapy in AIDS virus infections. Nat Med. 2003;9(7):861-866.

18. Quigley M, et al. Transcriptional analysis of HIVspecific CD8(+) $\mathrm{T}$ cells shows that PD- 1 inhibits $\mathrm{T}$ cell function by upregulating BATF. Nat Med. 2010; 16(10):1147-1151.

19. Speiser DE, et al. Rapid and strong human CD8+ $\mathrm{T}$ cell responses to vaccination with peptide, IFA, and $\mathrm{CpG}$ oligodeoxynucleotide 7909. J Clin Invest. 
2005;115(3):739-746

20. Zippelius A, et al. Effector function of human tumor-specific $\mathrm{CD}^{+} \mathrm{T}$ cells in melanoma lesions: a state of local functional tolerance. Cancer Res. 2004; 64(8):2865-2873

21. Speiser DE, et al. Memory and effector $\mathrm{CD}^{+} \mathrm{T}$ cell responses after nanoparticle vaccination of melanoma patients. J Immunother. 2010;33(8):848-858.

22. Rosenberg SA, Dudley ME. Adoptive cell therapy for the treatment of patients with metastatic melanoma. Curr Opin Immunol. 2009;21(2):233-240.

23. Sakuishi K, Apetoh L, Sullivan JM, Blazar BR, Kuchroo VK, Anderson AC. Targeting Tim-3 and PD-1 pathways to reverse T cell exhaustion and restore anti-tumor immunity. J Exp Med. 2010; 207(10):2187-2194.

24. Ahmadzadeh $M$, et al. Tumor antigen-specific $\mathrm{CD}^{+} \mathrm{T}$ cells infiltrating the tumor express high levels of PD-1 and are functionally impaired. Blood. 2009;114(8):1537-1544.

25. Appay $V$, et al. New generation vaccine induces effective melanoma-specific CD8+ T cells in the circulation but not in the tumor site. J Immunol. 2006; 177(3):1670-1678.

26. Choi S, Schwartz RH. Molecular mechanisms for adaptive tolerance and other $\mathrm{T}$ cell anergy models. Semin Immunol. 2007;19(3):140-152.

27. Derre L, et al. BTLA mediates inhibition of human tumor-specific CD8 $+\mathrm{T}$ cells that can be partially reversed by vaccination. J Clin Invest. 2010; 120(1):157-167.

28. Appay V, et al. Sensitive gene expression profiling of human $T$ cell subsets reveals parallel post-thymic differentiation for CD4+ and CD8+ lineages. J Immunol. 2007;179(11):7406-7414.

29. Critchley-Thorne RJ, Yan N, Nacu S, Weber J, Holmes SP, Lee PP. Down-regulation of the interferon signaling pathway in $\mathrm{T}$ lymphocytes from patients with metastatic melanoma. PLoS Med. 2007;4(5):e176.

30. Cham CM, Xu H, O'Keefe JP, Rivas FV, Zagouras P, Gajewski TF. Gene array and protein expression profiles suggest post-transcriptional regulation during CD8+ T cell differentiation. J Biol Chem. 2003; 278(19):17044-17052.

31. Willinger T, Freeman T, Hasegawa H, McMichael AJ, Callan MF. Molecular signatures distinguish human central memory from effector memory $\mathrm{CD}^{+} \mathrm{T}$ cell subsets. J Immunol. 2005;175(9):5895-5903.

32. Bengsch B, et al. Coexpression of PD-1, 2B4, CD160 and KLRG1 on exhausted HCV-specific CD8+ T cells is linked to antigen recognition and $\mathrm{T}$ cell differentiation. PLoS Pathog. 2010;6(6):e1000947.

33. Blackburn SD, et al. Coregulation of CD8+ T cell exhaustion by multiple inhibitory receptors during chronic viral infection. Nat Immunol. 2009 10(1):29-37.

34. Jin HT, et al. Cooperation of Tim- 3 and PD- 1 in CD8 ${ }^{+}$ T cell exhaustion during chronic viral infection. Proc Natl Acad Sci U S A. 2010;107(33):14733-14738.

35. Rey J, et al. The co-expression of 2B4 (CD244) and CD160 delineates a subpopulation of human CD8+ $T$ cells with a potent CD160-mediated cytolytic effector function. Eur J Immunol. 2006;36(9):2359-2366.

36. Fourcade J, et al. Upregulation of Tim-3 and PD-1 expression is associated with tumor antigen-specific CD8+ T cell dysfunction in melanoma patients. J Exp Med. 2010;207(10):2175-2186.

37. McMahan RH, et al. Tim-3 expression on PD-1+ $\mathrm{HCV}$-specific human CTLs is associated with viral persistence, and its blockade restores hepatocyte-directed in vitro cytotoxicity. J Clin Invest. 2010;120(12):4546-4557.

38. Macian F, Garcia-Cozar F, Im SH, Horton HF, Byrne MC, Rao A. Transcriptional mechanisms underlying lymphocyte tolerance. Cell. 2002;109(6):719-731.

39. Parish IA, et al. The molecular signature of CD8+
T cells undergoing deletional tolerance. Blood. 2009;113(19):4575-4585.

40. Willimsky G, Blankenstein T. Sporadic immunogenic tumours avoid destruction by inducing $T$ cell tolerance. Nature. 2005;437(7055):141-146.

41. Willimsky G, et al. Immunogenicity of premalignant lesions is the primary cause of general cytotoxic T lymphocyte unresponsiveness. J Exp Med. 2008; 205(7):1687-1700.

42. MillerJD, et al. Human effector and memory CD8+T cell responses to smallpox and yellow fever vaccines. Immunity. 2008;28(5):710-722

43. Gaucher D, et al. Yellow fever vaccine induces integrated multilineage and polyfunctional immune responses. J Exp Med. 2008;205(13):3119-3131.

44. Barbey C, et al. IL-12 controls cytotoxicity of a novel subset of self-antigen-specific human CD28+ cytolytic T cells. JImmunol. 2007;178(6):3566-3574.

45. Lienard D, et al. Ex vivo detectable activation of Melan-A-specific T cells correlating with inflammatory skin reactions in melanoma patients vaccinated with peptides in IFA. Cancer Immun. 2004;4:4.

46. Romero P, et al. Ex vivo staining of metastatic lymph nodes by class I major histocompatibility complex tetramers reveals high numbers of antigen-experienced tumor-specific cytolytic T lymphocytes. J Exp Med. 1998;188(9):1641-1650.

47. Hadrup SR, et al. Parallel detection of antigen-specific $\mathrm{T}$ cell responses by multidimensional encoding of MHC multimers. Nat Methods. 2009;6(7):520-526.

48. Roederer M, Nozzi JL, Nason MC. SPICE: Exploration and analysis of post-cytometric complex multivariate datasets. Cytometry A. 2011;79(2):167-174.

49. Subramanian A, et al. Gene set enrichment analysis: a knowledge-based approach for interpreting genome-wide expression profiles. Proc Natl Acad Sci US A. 2005;102(43):15545-15550. 